\title{
FACTORES DE INSERCIÓN AL DELITO DE TRÁFICO DE DROGAS EN LAS INTERNAS DEL CENTRO PENITENCIARIO DE MUJERES DE CONCEPCIÓN-JUNÍN
}

\section{INSERTING THE CRIME FACTORS OF DRUG TRAFFICKING IN INTERNAL CENTER PRISON WOMEN'S CONCEPTION - JUNÍN}

\author{
Marisol Condori Apaza'; Gustavo Alberto Reyna Arauco²
}

\section{RESUMEN}

En el Perú, así como en otros países, los delitos cometidos por mujeres se vienen incrementando cada vez más. La modernización y la mentada "equidad de género" no sólo se visualizan en la inserción de la mujer al ámbito público, sino también en su inserción a cometer delitos, lo cual indica que, el delito va perdiendo el "casi" exclusivo carácter masculino que socialmente se tenía adjudicado. De las actividades delictivas señaladas líneas arriba, un significativo porcentaje, en diversos penales de mujeres en el Perú, es el tráfico de drogas, ya que esta ilícita actividad les permite a muchas mujeres continuar con el desempeño de su rol de madre, esposa, abuela y ama de casa. En varias ocasiones son ellas quienes tienen a su cargo la venta de drogas proveídas por los varones, ya que son las menos sospechosas de infracción o simplemente por motivos de sobrevivencia, porque la mencionada actividad les permite sustentar los gastos de alimentación de su familia. En tal sentido, la investigación tuvo como objetivo conocer los factores de inserción al delito de tráfico de drogas. La investigación fue básica, con nivel descriptivo explicativo, diseño de investigación no experimental transversal y enfoque metodológico cuantitativo. La recolección de datos se realizó mediante un cuestionario estructurado aplicado a 24 internas por el delito de tráfico de drogas del Centro Penitenciario de Mujeres de Concepción - Junín. Los principales resultados hallados en la investigación indican que los factores sociales que influyeron en la inserción al delito de tráfico de drogas en las internas del Centro Penitenciario de Mujeres de Concepción - Junín son: el 75\% de internas pertenecieron a familias desintegradas y el 70,8\% a familias disfuncionales; el 100\% de internas manifestaron que su proceso de socialización fue inadecuado. El factor económico que influyó en la inserción al delito es la situación socioeconómica desfavorable, ya que, el 100\% manifestó haber incursionado en el delito por necesidad económica. Considerando el factor cultural, los resultados revelan que el $70,8 \%$ incursionaron en el delito obedeciendo a su mentalidad facilista y el $75 \%$ a su mentalidad ambiciosa para satisfacer sus necesidades.

Palabras clave: Delito, tráfico de droga, socialización, situación socioeconómica, mentalidad facilista y ambiciosa.

\section{ABSTRACT}

In Peru, as well as in other countries, crimes committed by women have been increasing more with time. Modernization and the so called "gender equity" are not only visualized in the insertion of women in the public sector, but also in their push to commit crimes, which indicates that the offense is losing the "almost" exclusive male character that socially was awarded. Among the criminal activities enumerated above, a significant percentage, in various criminal women penitentiaries in Peru, is the traffic of drugs, since this illicit activity allows many women to continue with the performance of their role of mother, wife, grandmother and housewife. On several occasions they are in charge of trading the drugs supplied by men, since they are the least infringement suspects or simply

1 Doctora en Sociología, Catedrática, consultora social e investigadora de la Universidad Nacional del Centro del Perú. marisol c a@hotmail.com

2 Magíster en Sociología, Catedrático e investigador de la Universidad Nacional del Centro del Perú. betreyna23@ hotmail.com 
for reasons of survival, because the above-mentioned activity enable them to sustain food expenses for your family. In this sense, the research had as objective knowing the factors of insertion to drug trafficking crime. The research was basic, with explanatory descriptive level, sectional non-experimental research design and quantitative methodological approach. The data collection was carried out using a structured questionnaire applied to 24 internal under charges of drug trafficking crime in the Women's Penitentiary Center Concepción- Junín. The main results found in the investigation indicate that social factors that influenced the insertion to the crime of drug trafficking in the inmates of the penitentiary for women of Conception - Junin are: $75 \%$ of inmates belonged to broken families and $70.8 \%$ to dysfunctional families; $100 \%$ of internal stated that the process of socialization was inadequate. The economic factor that influenced the insertion to crime is the unfavourable socio-economic situation, since the 100\% stated that they had made inroads in the crime by economic necessity. Considering the cultural factor, the results reveal that the $70.8 \%$ got into crime in obedience to their facile mentality and $75 \%$ to their mentality ambitious to meet their needs.

Key Words: crime, drug trafficking, socialization, socio-economic situation, facile mentality and ambitious.

\section{INTRODUCCIÓN}

Investigar la problemática "mujer y delito", nos conlleva a reflexionar sobre la forma en la cual el rol de ésta fue percibido por la sociedad. En aspectos morales fue considerada superior al varón; pero, en términos biológicos fue considerada débil y sumisa. Por ende, fue difícil concebir la idea de la mujer agresiva y delincuente y, frente a la existencia de casos aislados, se la consideraba como un ser con perturbaciones psicológicas; planteamiento que no responde a la realidad, sino que el delito en la mujer obedece a factores sociales, económicos y culturales. Por otro lado, la situación en la que se encuentran las mujeres privadas de su libertad es degradante. En tal sentido la presente investigación formuló el problema: ¿Qué factores influyeron en la inserción al delito de tráfico de drogas por parte de las internas del Centro Penitenciario de Mujeres de Concepción - Junín?

Frente a esta problemática, la investigación tuvo como objetivos: Identificar y analizar los principales factores que influyeron en la inserción al delito de tráfico de drogas por parte de las internas del Centro Penitenciario de Mujeres de Concepción - Junín.

La investigación se justifica porque el problema estudiado es de importancia para las ciencias sociales, en la medida que en el Perú, así como en el departamento de Junín, los delitos cometidos por parte de las mujeres se vienen incrementando cada vez más y, hasta la actualidad no existen suficientes estudios que aborden la realidad y problemática penitenciaria de la mujer. En tal sentido, la presente investigación tiene por finalidad abordar la problemática en mención desde un enfoque de género en el ámbito de la sociología del delito.

Las diversas teorías sociológicas que sustentan la investigación, manifiestan que el comportamiento humano delictivo es influido en gran medida por el contexto social en el que se desenvuelve el individuo. Así tenemos a la teoría funcionalista, la que sustenta que la persona que incurre en delito es desviada, y las altas tasas de desviación son el resultado de la anomía, una condición de "anormalidad" o pérdida de las reglas sociales aceptadas dentro de una sociedad. La teoría del control, argumenta que la mayoría de las personas son tentadas a cometer de vez en cuando un comportamiento prohibido, cuando los grupos sociales donde se interrelaciona (familias, amigos y vecinos), no están involucrados en actividades socialmente aceptadas (como escuela y trabajo), y más fuerte es su creencia en las oportunidades legítimas y por tanto es más probable aceptarlas. La teoría de la transmisión social manifiesta que los individuos se vuelven delincuentes o criminales debido a la asociación diferencial, es decir, cuando son expuestos a más normas y valores prodelictivos que antidelictivos por periodos largos o cuando se encuentran en 
situaciones que premian el comportamiento delictivo. Los individuos adquieren actitudes de la gente con la que se asocian; es decir, de las influencias "buenas" o "malas". La teoría interaccionista señala que la desviación social se aprende mediante la interacción con los demás, vinculan el delito y el crimen con la asociación diferencial. Señala que en una sociedad donde existen diversas subculturas, algunos ambientes sociales tienden a orientar a los individuos hacia actividades ilegales y otros no. La teoría del etiquetaje señala que la etiqueta que dan algunos individuos a otros sobre una conducta desviada puede empujar a la reiteración de este tipo de conducta. La teoría del conflicto establece la existencia de una estrecha relación entre poder y desviación social. Señala que las normas no son neutrales, sino que son instrumentos que los poderosos emplean para mantener su situación de privilegio y el orden social, estas favorecen a los más privilegiados, a los ricos que detentan el poder y que el privilegiado cuenta con más recursos para evitar su condena. Indica que las leyes no son buenas y naturales. El poder y sus instituciones usan etiquetas contra los que critican y amenazan su poder, por ejemplo a los que amenazan la propiedad privada los tildan de ladrones, a los que no pueden 0 quieren trabajar de vagos, a los que no respetan la autoridad de violentos.

Se presenta la investigación esperando aportar al conocimiento de las ciencias sociales respecto a la problemática mujer y delito en el interior del país.

\section{METODOS Y MATERIALES}

El tipo de investigación es básica; con nivel descriptivo explicativo, ya que la investigación en mención pretende describir y explicar las variables de investigación y la relación que existe entre estas a fin de dar cuenta los factores de inserción al delito y la situación actual que afrontan las internas del Centro Penitenciario de Mujeres de Concepción - Junín. El diseño de la investigación es no experimental transversal, teniendo en cuenta el enfoque cuantitativo.

La recolección de datos se realizó mediante un cuestionario estructurado aplicado a 24 internas por el delito de tráfico de drogas del Centro Penitenciario de Mujeres de Concepción - Junín.

\section{RESULTADOS}

\section{Factores que influenciaron en la inserción al delito}

Las internas por tráfico de drogas que purgan condena en el Centro Penitenciario de Mujeres de ConcepciónJunín, incursionaron en el delito influenciadas por diversos factores, tales como: pertenencia a familias desintegradas y disfuncionales, proceso de socialización inadecuada, situación socioeconómica desfavorable, mentalidad facilista y ambiciosa. Los mismos que se presentan a continuación.

\section{A.Pertenencia a familias desintegradas y disfuncionales}

Considerando que la familia es la unidad básica de la sociedad, en la cual sus miembros van a obtener la base del proceso de socialización, es necesario considerar la composición en cuanto a miembros pertenecientes a sus familias y las funciones que cumplían los padres de las internas por delito de tráfico de drogas en el Centro Penitenciario de Mujeres de Concepción - Junín. Tal es así que, el $75 \%$ indicó haber pertenecido a familias desintegradas y el 70,8\% a familias disfuncionales, tal como se muestra en los gráficos 1 y 2.

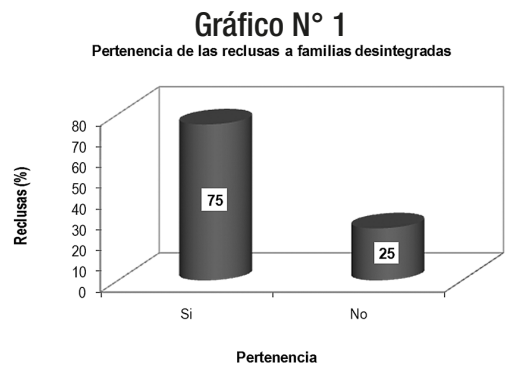

Fuente: Encuesta aplicada a las internas del CPMC-J

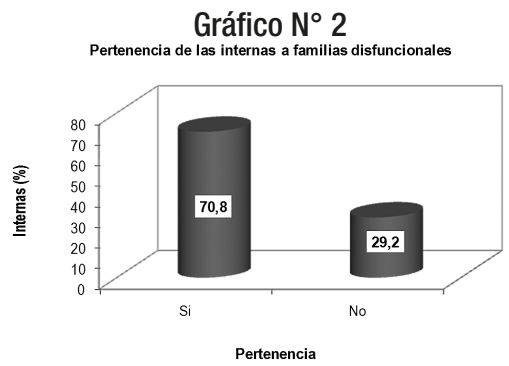

Fuente: Encuesta aplicada a las internas del CPMC-J 


\section{B. Socialización inadecuada}

La socialización es el "proceso a través del cual un individuo aprende normas, valores, actitudes y comportamientos "adecuados"y así, consecuentemente, pasa a ser miembro de un grupo, una sociedad 0 una cultura. La socialización ocurre a través de la interacción social. Si bien dura toda la vida, el proceso de socialización es más intenso durante la infancia a través de la institución familiar (socialización primaria), continuando luego en la edad adulta a través de distintas instituciones sociales (socialización secundaria) (1). Los resultados revelan que el $100 \%$ de internas tuvieron un proceso de socialización inadecuado y este fue un factor significativo que influyó para la incursión al delito.

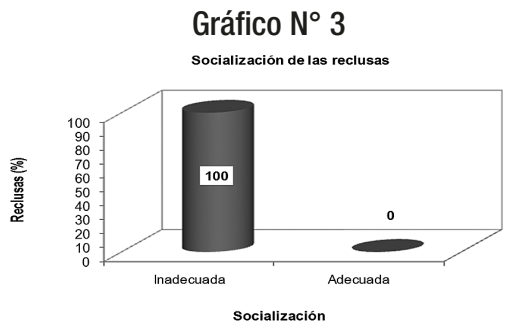

\section{Situación socioeconómica desfavorable}

La situación socioeconómica que atraviesan muchas mujeres es un factor que influye en la incursión al delito de tráfico de drogas. Merton (2) manifestó que "la desviación es un producto del sistema social, no de anormalidad individual. Las personas se enrolan en la desviación cuando una cultura estimula apetitos que no pueden ser satisfechos". Las internas del Centro Penitenciario de Mujeres de Concepción - Junín, en un $100 \%$ manifestaron que incursionaron al delito de tráfico de drogas por necesidad económica, tal como se muestra en el gráfico 4.

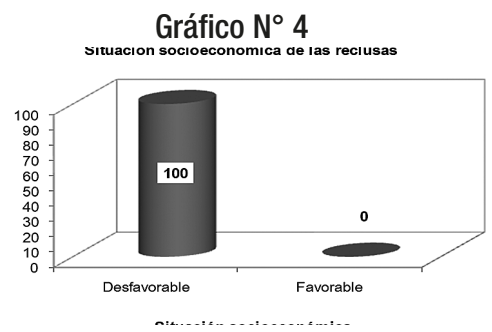

Fuente: Encuesta aplicada a las internas del CPMC-J

\section{Mentalidad facilista y ambiciosa de las internas}

La mentalidad que tienen las personas depende de la cultura y la visión que tengan frente a la vida. Mientras que para unos la vida debe ser digna y para ello deben esforzarse por obtener los medios necesarios y lograr una vida de calidad, para otros en cambio, la obtención de esos medios no considera esfuerzo, es por ello que se dedican a actividades ilícitas y fáciles para obtener dinero. En tal sentido, la mentalidad facilista y ambiciosa influyó para que las internas del Centro Penitenciario de Mujeres de Concepción-Junín, incursionen en el tráfico de drogas; ya que, la mayoría de las internas $(70,8 \%)$ consideran que la venta de drogas era una manera fácil de obtener dinero, y el $75 \%$ manifestó que ambicionaba tener otros logros, que con un trabajo decente no se podía lograr.

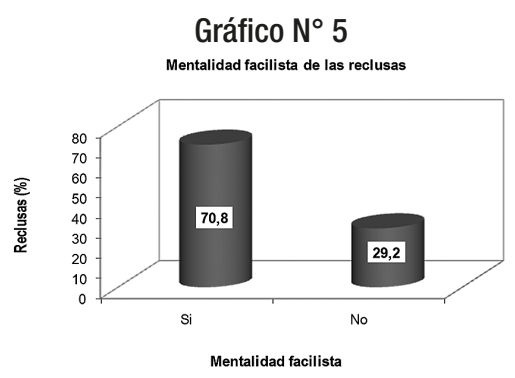

Fuente: Encuesta aplicada a las internas del CPMC-J

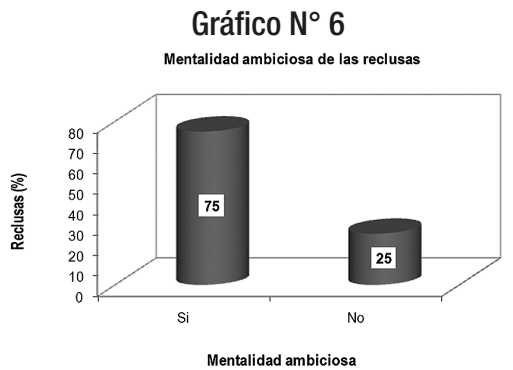

Fuente: Encuesta aplicada a las internas del CPMC-J

\section{DISCUSIÓN DE RESULTADOS}

Los factores que influyen en la inserción al delito son diversos y como tal también han surgido diversas teorías y estudios que lo explican, los cuales se clasifican en "individuales, biológicos y sociales 0 ambientales" (3) (4) (5). Sin embargo, a pesar de la 
existencia de una variedad de trabajos que intentan explicar la influencia de los factores individuales y biológicos del origen de la delincuencia, la mayoría no logran datos incuestionables. Por lo que concordamos con los planteamientos de Delgado (6), Valverde (7) y, Wilson y Howell (5) al aseverar que:

"(...) los factores psicobiológicos pueden estar influyendo en el origen de los actos delictivos, pero se hace necesaria la presencia de otros factores para llegar a conocer claramente el comportamiento delictivo. Parece claro, por 10 tanto, que la presión de -los factores sociales o- del medio ambiente es fundamental en la génesis de la delincuencia. Es decir, el contexto, si tiene cargas delictógenas, puede ser no sólo influyente 0 favorecedor de la criminalidad, sino desencadenante, ya que, no debemos olvidar, que los comportamientos son aprendidos y no heredados y los individuos crecen y se desarrollan recibiendo cargas positivas y negativas del ambiente que les rodea De todos estos factores, los más influyentes en el desarrollo de la conducta delictiva, y por tanto, más estudiados e investigados son la familia, la escuela y la ocupación laboral."

Considerando que un delito es "la violación de una norma que ha sido codificada en una ley y es respaldada por el poder y la autoridad del Estado" (8), es necesario conocer los factores que influyen en una persona para que cometa un delito, y más aún si es cometido por una mujer, ya que diversos estudios dan a conocer que en las dos últimas décadas, los índices de mujeres privadas de su libertad se ha incrementado significativamente, I0 cual tiene que ver sobre todo con delitos de consumo y tráfico de drogas tanto en países desarrollados como en vías de desarrollo incluyendo a Perú, país en el cual la tendencia de mujeres encarceladas va ascendiendo año a año. Tal es el caso de la región Junín, que cuenta con un Centro Penitenciario de Mujeres, en la provincia de Concepción, cuya población encarcelada va en ascenso y, por otro lado, del total (56 internas), la mayoría (24 internas) está privada de su libertad por el delito de tráfico ilícito de drogas. De acuerdo a los resultados obtenidos, los factores sociales que influenciaron para que las mismas cometan este delito son: la pertenencia a familias desintegradas y disfuncionales, proceso de socialización inadecuado, condiciones socioeconómicas desfavorables, mentalidad facilista y ambiciosa, resultados que a continuación se discuten y analizan.

Considerando a la desintegración familiar como el rompimiento de la unidad familiar, los resultados del cuestionario aplicado a las internas del Centro Penitenciario de Concepción-Junín, nos revelan que el 75\% de internas pertenecieron a familias desintegradas. Así también el $70.8 \%$ integraron e integran familias disfuncionales. Estos datos dan pie a lo estudiado por Gottfredson y Hirschi, quienes concluyeron que "(...) entre más unidas estén las personas a las familias, (...) más involucradas están en actividades socialmente aceptadas. (9)

Entonces, las funciones familiares son fundamentales para la conservación de la sociedad y de su equilibrio, ya que el ser humano tiene la necesidad de un cierto aprendizaje y de una adaptación social para su supervivencia. Diversas investigaciones (10) han demostrado que:

"(...) al interior de la familia se plantea también el problema de la prevención o la facilidad de la conducta delictiva. Entre los factores familiares negativos señalados como determinantes de la delincuencia se encuentran: las malas relaciones familiares, falta de vigilancia parental, la disociación del grupo familiar, las carencias afectivas familiares, entre otros."

Un segundo factor considera que el 100\% de internas manifestó que tuvo un proceso de socialización inadecuado, entendiendo que la socialización es el proceso mediante el cual el individuo aprende normas, valores, actitudes y comportamientos "adecuados" para que pase a ser miembro bien integrado de un grupo, una sociedad o una cultura. En tal sentido, las personas con características desviadas y delictivas, son aquellas que tuvieron problemas de socialización e integración en los diversos agentes como la familia, escuela y comunidad. En cierta medida aquellas personas se sienten excluidas socialmente, y de acuerdo con Martín (11):

"(...) asumen valores, pautas conductuales y formas de vida diferentes a los del grupo social normalizado, lo que lleva al acrecentamiento de las diferencias de un grupo y otro, y a un clima de 
violencia, malestar social y delincuencia."

Quedando claro entonces que, del proceso de socialización depende que los individuos sean miembros bien integrados de la sociedad o simplemente sean personas con comportamientos desviados 0 delictivos. En concordancia con ello, la teoría psicológica sustenta que, la conducta desviada y el delito son el resultado de una socialización fallida más que de factores hereditarios.

Por otro lado, la teoría del control, hace referencia a la capacidad de autodominio que no todas las personas tienen, esto por ser parte de una socialización defectuosa, y refiriéndose a la familia agrega que el control paternal inadecuado lleva a un inadecuado autodominio." (12)

En la misma línea, la teoría de la transmisión cultural percibe a la desviación como el resultado de la socialización a una subcultura que aplaude actitudes y comportamientos que la corriente cultural principal rechaza. Precisamente, Sutherland (13) señala que:

(...) los individuos se vuelven delincuentes 0 criminales debido a la asociación diferencial, es decir, cuando son expuestos a más normas y valores prodelictivos que antidelictivos por periodos largos o cuando se encuentran en situaciones que premian el comportamiento delictivo (...) Los individuos adquieren actitudes de la gente con la que se asocian; es decir, de las influencias "buenas" o "malas".

Apoyando a lo manifestado, los sociólogos interaccionistas señalan que la desviación social se aprende mediante la interacción con los demás quienes-vinculan el delito y el crimen con la asociación diferencial. Señalan además que en una sociedad donde existen diversas subculturas, algunos ambientes sociales tienden a orientar a los individuos hacia actividades ilegales y otros no.

Anthony Giddens, en la misma perspectiva, manifiesta que:

"Los individuos se vuelven delincuentes al asociarse con los portadores de normas delictivas. El comportamiento delictivo se aprende en grupos primarios y especialmente entre compañeros (...) las acciones delictivas son tan aprendidas como las que respetan la ley. Los ladrones intentan ganar dinero al igual que la gente al igual que la gente que tiene trabajos convencionales" (14)

Diversos estudios sustentan que la mayoría de mujeres privadas de su libertad provienen de sectores socioeconómicos socialmente desfavorecidos y en situaciones de marginalidad, lo cual cuantiosas veces genera actos desviados y/o delictivos tales como: el tráfico ilícito de drogas, el pandillaje, la prostitución, el robo, entre otros.

Un tercer factor de inserción al delito es la situación socioeconómica desfavorable, ya que los resultados revelan que, el 100\% de internas por tráfico ilícito de drogas del CPMC-J, incursionaron en tal delito por necesidad económica. En tal sentido, coincidiendo con M. Romero y R. Aguilera:

"(...) cada día mayor número de mujeres se ve involucrada en la comisión de actos violentos y delincuencia y que la mayoría de las mujeres encarceladas proviene de sectores de la población económica y socialmente desfavorecida y que permanecen encarceladas por delitos típicos de personas que han vivido en la pobreza y que han sido violentadas la mayor parte de sus vidas." (15)

Asimismo, los planteamientos de la teoría funcionalista, refieren que, el individuo económicamente más débil tiene que optar por conformarse con lo que tiene o termina delinquiendo para conseguir aquello que para él es inalcanzable por otros medios. Los valores dominantes de las sociedades se reducen cada vez más a la competitividad y el logro del éxito. Hay que conseguir dinero, propiedades y estatus social sin importar tanto los medios empleados para conseguir esos fines. Pero ocurre que las clases bajas y más desfavorecidas, los grupos minoritarios, no se hallan en el mismo punto de partida para acceder a esta lucha que se presenta desigual. Entonces las personas con menos recursos sufren tensiones porque desean alcanzar unos fines que les son inalcanzables. Su disconformidad con las normas imperantes generan las tensiones que conducen a optar por un comportamiento delictivo como mejor solución para alcanzar el estatus anhelado. 
"Según Merton, en un momento en que el conjunto de sociedades se está haciendo más rica la pregunta seria ¿por qué sigue aumentando los índices de delincuencia? Al subrayar el contraste existente entre el aumento de las aspiraciones y la persistencia de las desigualdades, Merton señala que "la sensación de privación relativa es un elemento importante a la hora de interpretar el comportamiento desviado." (16)

Existe una desarticulación entre lo que la sociedad propone y los medios que esa misma sociedad pone a disposición de sus miembros para alcanzar dichos fines. Lo que da pie a demostrar que el comportamiento desviado es el resultado de las propias estructuras sociales. Tal circunstancia afecta a las clases bajas y desfavorecidas con una mayor intensidad, ya que parten de una situación más desigual para intentar ganar niveles en sus estatus respectivos. Cuando estos individuos se dan cuenta de sus carencias y de su imposibilidad de alcanzar y disfrutar bienes materiales que la sociedad pone ante sus ojos, éstos desde su situación económica delinquen sin reparo hasta conseguir un nivel económico superior.

Esta es la explicación que da Merton al fenómeno de la delincuencia. Y sería quizás también explicativo de por qué la delincuencia se da más en las clases más desfavorecidas: porque dichas clases se hallan, como decíamos, en una situación menos favorable para acceder a determinados bienes y posiciones sociales. La clase alta necesitaría delinquir menos, puesto que goza de un bienestar material que sirve como disuasor de conductas divergentes, aunque bien es verdad que la ambición del ser humano por poseer más y más no conoce límites. (17)

Por otra parte, la teoría del conflicto refiere que:

“(...) los individuos optan deliberadamente por implicarse en comportamientos desviados para responder a comportamientos del sistema capitalista (...) Sitúan el análisis del delito y de la desviación en el marco de la estructura social y de la perseverancia del poder por parte de la clase dominante" (17)

Los mismos teóricos señalan además que, la desviación y los delitos tienen lugar en todos los niveles sociales y que deben enmarcarse dentro del contexto social de las desigualdades existentes entre los diferentes grupos y en sus intereses contrapuestos. Denuncian que a los poderosos (delincuentes de cuello blanco) que quebrantan la legalidad, no se les sanciona, como si lo hacen con los delincuentes de la clase media, sectores bajos y débiles de la sociedad (prostitutas, toxicómanos, ladronzuelos, entre otros.)

Sumándose a tal planteamiento, Guelles y Ann , señalan que:

"Aunque Marx no desarrolló de manera extensa este tema, percibió el delito como derivado de la división de la sociedad capitalista en dos clases separadas y desiguales: los dueños de los medios de producción y los obreros. Para simplificar un poco, los pobres cometen delitos para obtener bienes materiales que se les han negado y para expresar su enojo y frustración. Los miembros de la clase gobernante también violan las normas para mantener su posición y privilegios; a diferencia de las masas, esta clase tiene el poder para prevenir que sus acciones no sean clasificadas como desviadas 0 delictivas. De esta manera la teoría del conflicto no se enfoca en los grupos que rompen las reglas, sino en los grupos que hacen las reglas." (8)

Entonces podemos afirmar que, encontrarse en una situación económica desfavorable influye para que las personas se inserten en actos desviados y/o delictivos, los cuales posteriormente tendrán sanciones formales 0 informales.

Finalmente se consideró el factor cultural, como un elemento que influye en la incursión al delito, específicamente se ha incluido la mentalidad de las internas, obteniendo como resultado que el $70.8 \%$ de internas manifestó haber incursionado al tráfico ilícito de drogas porque consideraban que era una manera fácil de obtener dinero. Desde la perspectiva sociocultural, es considerada como mentalidad facilista que hace referencia a individuos que son parte de una "cultura" que va trastocando su mentalidad, ya que en vez de fomentar una ética de trabajo, esfuerzo, ahorro y progreso, van fomentando ideas facilistas cuya tendencia es optar por la vía más fácil la consecución de algún objetivo. 
Precisamente diversos problemas que aquejan nuestra sociedad, tales como la delincuencia, prostitución, el tráfico ilícito de drogas, entre otros, "son muestras significativas de este fenómeno ligado a una mentalidad que ha recibido el adjetivo de "plástica"” (18).

En la sociedad peruana, así como en algunas otras, este tipo de mentalidades persiste porque la sociedad está dividida entre quienes censuran estas mentalidades y sus posteriores acciones y, quienes las protegen. Al respecto Edwin Sutherland señaló que:

"...en una sociedad heterogénea existen diversos grupos, cada uno con su propio juego de normas... Algunos premian el esfuerzo y trabajo duro; otros defienden "la vida fácil". (13)

Por otro lado, la mentalidad facilista está asociada a la mentalidad ambiciosa, tal es así que el 75\% de internas del PMC-J, se caracterizan por tener una mentalidad ambiciosa. Tal mentalidad incita a muchos individuos a corromperse y cometer actos ilícitos con la finalidad de obtener cada vez mayor dinero y posición social.

Estas ansias de obtener logros, nace de una imponente cultura del consumo que se va implantando cada vez más en la mentalidad del individuo, pero no todos cuentan con los recursos para poder satisfacer necesidades, deseos 0 ambiciones; en estas circunstancias algunos se ven presionados a realizar actos delictivos. Consolidando este planteamiento Merton manifiesta que:

"...es la sociedad de consumo la que impulsa a los individuos a la búsqueda desaforada del éxito monetario. El dinero todo lo puede y con dinero todo se compra. Aquellos a quienes la sociedad no ofrece oportunidades para acceder a los niveles de bienestar sugeridos y deseados, se verán totalmente presionados a cometer delitos, encaminados a conseguir las metas codiciadas. En consecuencia, la anomia no es propiamente una situación de crisis debida a factores coyunturales, sino una disfunción estructural, crónica, endémica e inherente a cierto modelo de sociedad. Dicha sociedad, crea en el individuo una presión tal que cuando se le hace insostenible se manifiesta a través de conductas desviadas, indeseables y muchas de las veces delictivas. En el fondo de todo ello late la frustración de querer tener un determinado status económico y no poder acceder a él; de querer poseer determinados bienes que se antojan inalcanzables para el nivel de renta disponible. La manera más fácil de obtener esos niveles de renta es a través de conductas delictivas. La sociedad no hace otra cosa que proponer al individuo unos objetivos pero sin proporcionarle los medios para alcanzar tales metas. Entonces el individuo o se conforma con lo que tiene -con su status- 0 delinque para alcanzar un nivel superior." (16)

\section{CONCLUSIONES}

1. Las familias a las que pertenecieron cuando niñas y adolescentes las internas por tráfico ilícito de drogas del CPMC-J, fueron consideradas desintegradas y disfuncionales. En tal sentido, la desintegración familiar como fenómeno con mayor impacto en la sociedad peruana implica la ruptura de la unidad familiar, la misma que traerá consigo diversos problemas de índole social, entre ellas la desviación social de sus miembros y la incursión en delitos.

2. La socialización como proceso a través del cual el individuo aprende normas, valores, actitudes y comportamientos "adecuados" para que pase a ser miembro bien integrado de la sociedad, no estuvo presente de la manera adecuada en las internas del CPMC-J, ya que en los tres principales agentes de socialización (familia, escuela y comunidad) éstas consideran que no aprendieron normas, ni valores suficientes.

3. Las internas por tráfico ilícito de drogas del CPMC-J, en su mayoría incursionaron en el delito por necesidad, ya que se encontraban en una situación económica desfavorable.

4. La mentalidad facilista y ambiciosa que tienen las internas frente a la vida es un factor fundamental que explica muchas situaciones, entre ellas el porqué de la incursión a actos delictivos.

\section{REFERENCIAS BIBLIOGRÁFICAS}

1. Del Acebo , R. Diccionario de Sociología, Claridad, Buenos Aires, 2006.

2. Merton, R. En: Guelles , R . Sociología con aplicaciones en países de habla hispana. McGrawHill// Interamericana Editores S.A., México, 2007. 
3. Farrigton, D.P. The development of offending and antisocial behavior from childhood: key findings from the Cambridge study in delinquent development. Journal of Child Psychology and Psychiatry, 1995.

4. Torrente, G. y Merlon, F. Aproximación a las características psicosociales de la delincuencia de menores en Murcia. Anuario de Psicología Jurídica, 1999.

5. Wilson, J.J. y Howell, J.C. (1995). Comprehensive strategy for serious, violent and chronic juvenile offenders. En D.G. Rojek y G.J. Jensen (Eds.), Exploring delinquency: causes and control Los Ángeles: Roxbury, 1995.

6. Delgado, S. Delito y drogodependencia. En: S. Delgado, E. Esbel, T. Rodríguez y J.L. González, Psiquiatría legal y forense (Vol. 2, pp. 555-589). Madrid: Colex, 1994.

7. Valverde, J. Seminario sobre las consecuencias de la prisión. Instituto Andaluz Interuniversitario de Criminología. Universidad de Sevilla, 1996.

8. Guelles, R. J. y Levine, A. Sociología con aplicaciones en países de habla hispana. Sexta Ed. Mc WrawHill/Interamericana Editores, S.A. de C. V.: México D.F., 2000.

9. Gottfredson, M. y Hirschi, T. Una teoría general del crimen. Stanford, C.A. Stanford, University Press, 1990.

10. Koudon, 0. Familles dissociès "secondaires". En: Cöte d'ivoire et comportements délinquants des adolescents. In: Revue International de Criminologie et de police technique, Vol. 47, No.2 Paris, 1989.
11. Martín, V. M. Actitudes de los internados en prisión, menores de veintiún años, ante la función reeducadora del medio penitenciario en el ámbito Andaluz. Departamento de Teoría e Historia de la Educación. Universidad de Malaga, S/F.

12. Hirschi, T . Causes of Delincuency. Berkeley, C.A: University of California Press, 1969.

13. Suthrland, E. White Collar Crime: The Uncut Version. New Waven, CT: Yale University Press, 1949.

14. Giddens, A. Sociología. Alianza Editorial. 4ta. Edición, Madrid - España A, 2002.

15. Romero, M. y Aguilera, R. ¿Por qué Delinquen las Mujeres? Parte I. Perspectivas Teóricas Tradicionales. Revista: Salud Mental Vol 25, No. 5, 2002.

16. Merton R. En: Giddens, A. Sociología. Alianza Editorial. 4ta. Edición, Madrid - España A, 2002.

17. Merton, R. En: Risueño, J. ¿Por qué existe la delincuencia? una aproximación a sus causas, 2009. En: www.icalba.com/pdf/DELINCUENCIA.doc (Recuperado el 25 de enero del 2012)

18. Pérez, G. J. Ética y moral de la comunicación. En: http://www.scribd.com/doc/2269366/Etica-yMoral-de-la- comunicacion+teorias+sociales+$\mathrm{de}+\mathrm{la}+$ mentalidad+facilista\&ct=clnk (Recuperado el 25 de enero del 2012) 